\title{
The high-mass star-forming region IRAS 18182-1433
}

\author{
H. Beuther ${ }^{1}$, Q. Zhang ${ }^{2}$, T. K. Sridharan ${ }^{2}$, C.-F. Lee ${ }^{2}$, and L. A. Zapata ${ }^{2,3}$ \\ 1 Max-Planck-Institute for Astronomy, Königstuhl 17, 69117 Heidelberg, Germany \\ e-mail: beuther@mpia-hd.mpg.de \\ 2 Harvard-Smithsonian Center for Astrophysics, 60 Garden Street, Cambridge, MA 02138, USA \\ e-mail: name@cfa.harvard.edu \\ 3 Centro de Radioastronomía y Astrofísica, UNAM, Apdo. Postal 3-72 (Xangari), 58089 Morelia, Michoacán, México
}

Received 20 January 2006 / Accepted 23 March 2006

ABSTRACT

\begin{abstract}
Aims. We present mm line and continuum observations at high spatial resolution characterizing the physical and chemical properties of the young massive star-forming region IRAS 18182-1433.

Methods. The region was observed with the Submillimeter Array in the $1.3 \mathrm{~mm}$ band. The data are complemented with short-spacing information from single-dish $\mathrm{CO}(2-1)$ observations. $\mathrm{SiO}(1-0)$ data from the VLA are added to the analysis.

Results. Multiple massive outflows emanate from the mm continuum peak. The $\mathrm{CO}(2-1)$ data reveal a quadrupolar outflow system consisting of two outflows inclined by $\sim 90^{\circ}$. One outflow exhibits a cone-like red-shifted morphology with a jet-like blue-shifted counterpart where a blue counter-cone can only be tentatively identified. The $\mathrm{SiO}(1-0)$ data suggest the presence of a third outflow. Analyzing the ${ }^{12} \mathrm{CO} /{ }^{13} \mathrm{CO}$ line ratios indicates decreasing $\mathrm{CO}$ line opacities with increasing velocities. Although we observe a multiple outflow system, the mm continuum peak remains single-peaked at the given spatial resolution ( 13 $500 \mathrm{AU})$. The other seven detected molecular species - also high-density tracers like $\mathrm{CH}_{3} \mathrm{CN}, \mathrm{CH}_{3} \mathrm{OH}, \mathrm{HCOOCH}_{3}$ - are all $\sim 1-2^{\prime \prime}$ offset from the mm continuum peak, but spatially associated with a strong molecular outflow peak and a $\mathrm{cm}$ emission feature indicative of a thermal jet. This spatial displacement between the molecular lines and the mm continuum emission could be either due to an unresolved sub-source at the position of the $\mathrm{cm}$ feature, or the outflow/jet itself alters the chemistry of the core enhancing the molecular abundances toward that region. A temperature estimate based on the $\mathrm{CH}_{3} \mathrm{CN}\left(12_{k}-11_{k}\right)$ lines suggests temperatures of the order of $150 \mathrm{~K}$. A velocity analysis of the high-density tracing molecules reveals that at the given spatial resolution none of them shows any coherent velocity structure which would be consistent with a rotating disk. We discuss this lack of rotation signatures and attribute it to intrinsic difficulties to observationally isolate massive accretion disks from the surrounding dense gas envelopes and the molecular outflows.
\end{abstract}

Key words. stars: formation - ISM: jets and outflows - ISM: molecules - stars: early-type - stars: individual: IRAS 18182-1433 stars: winds, outflows

\section{Introduction}

The formation of massive stars is an important topic by itself, but it is also essential for understanding cluster formation and the Galactic evolution. Significant observational progress has been made over the last decade by statistical studies of large samples of young massive star-forming regions (e.g., Plume et al. 1992, 1997; Molinari et al. 1996, 1998, 2000; Hunter et al. 2000; Sridharan et al. 2002; Beuther et al. 2002a-c; Mueller et al. 2002; Shirley et al. 2003; Walsh et al. 2003; Faúndez et al. 2004; Hill et al. 2005; Klein et al. 2005). However, to really understand the physical properties at the inner center of the massive starforming regions at very early evolutionary stages, high-spatialresolution studies at (sub)mm wavelengths are a more adequate way of investigation. Observational studies of various massive star-forming regions with different interferometers have started disentangling the complex nature of high-mass star formation (e.g., Cesaroni et al. 1997; Zhang et al. 1998; Wyrowski et al. 1999; Shepherd et al. 2000; Beuther et al. 2005b; Gibb et al. 2003; Garay et al. 2003; Su et al. 2004). Nevertheless, we are still at the beginning of such studies, and there is urgent need to increase the number of investigated sources at high spatial and spectral resolution to base the conclusions on a more statistical foundation. To mention a few specific questions we are interested to tackle: what is the nature of massive molecular outflows, in an evolutionary sense as well as between sources of different mass and luminosity? What is the core mass distribution at the earliest evolutionary stages, can we infer information about the formation of the Initial Mass Function? What is the nature of potentially embedded accretion disks, and which way to best investigate them? Here, we present Submillimeter Array $\left(\mathrm{SMA}^{1}\right) 1.3 \mathrm{~mm}$ continuum and line observations toward the young high-mass star-forming region IRAS 18182-1433 addressing some of these questions.

The source IRAS $18182-1433$ is part of an intensely studied sample of 69 potential High-Mass Protostellar Objects (HMPOs) at early evolutionary stages prior to forming a significant UCHII region (Sridharan et al. 2002; Beuther et al. $2002 \mathrm{a}-\mathrm{c}$ ). At a near kinematic distance of $4.5 \mathrm{kpc}$, the luminosity and gas mass from the region are $\sim 10^{4.3} L_{\odot}$ and $\sim 1500 M_{\odot}$, respectively (Sridharan et al. 2002; Beuther et al. 2002a). The region harbors a bipolar outflow with an outflow mass of $\sim 30 \mathrm{M}_{\odot}$ and an outflow energy of $\sim 2.3 \times 10^{46} \mathrm{erg}$ (Beuther et al. 2002b). Furthermore, IRAS 18182-1433 shows OH, Class II $\mathrm{CH}_{3} \mathrm{OH}$, and $\mathrm{H}_{2} \mathrm{O}$ maser emission (Forster \& Caswell 1999;

1 The Submillimeter Array is a joint project between the Smithsonian Astrophysical Observatory and the Academia Sinica Institute of Astronomy and Astrophysics, and is funded by the Smithsonian Institution and the Academia Sinica. 
Walsh et al. 1998; Beuther et al. 2002c) but was not detected in $\mathrm{cm}$ continuum emission down to $1 \mathrm{mJy}$, indicating the early evolutionary stage of the source (Sridharan et al. 2002). However, recent sensitive VLA observations at $\mathrm{cm}$ and $7 \mathrm{~mm}$ wavelengths detected two weak $\mathrm{cm}$ and one $7 \mathrm{~mm}$ continuum source in this region (Zapata et al. 2005). A spectral index analysis of the three sub-sources shows that the $7 \mathrm{~mm}$ emission likely traces the main massive protostellar object in the field, whereas one $\mathrm{cm}$ feature is consistent with a thermal jet $\sim 2^{\prime \prime}$ offset from the $7 \mathrm{~mm}$ peak. The other $\mathrm{cm}$ feature $\sim 11^{\prime \prime}$ south-east of the $7 \mathrm{~mm}$ peak shows signatures of non-thermal synchrotron emission (Zapata et al. 2005, see also Fig. 2). De Buizer et al. (2005) observed this region in the mid-infrared at $10.5 \mu \mathrm{m}$ and $18.1 \mu \mathrm{m}$ wavelength. They detected a weak mid-infrared source close to the $7 \mathrm{~mm}$ and the $\mathrm{cm}$ thermal jet feature. Furthermore, they detected another far stronger and extended mid-infrared source $\sim 10^{\prime \prime}$ toward the south-east which appears to be associated with the cm continuum emission with negative spectral index (Zapata et al. 2005).

\section{Observations}

\subsection{Submillimeter Array observations}

The HMPO IRAS 18182-1433 was observed with the Submillimeter Array (SMA) on April 30th 2004 with 6 antennas at $1.3 \mathrm{~mm}$ in the compact configuration with projected baselines between 9.8 and $53 \mathrm{k} \lambda$. The phase center was RA $=18^{\mathrm{h}} 21^{\mathrm{m}} 09.098^{\mathrm{s}}$, Dec $=-14^{\circ} 31^{\prime} 49^{\prime} .29$ (J2000.0) with a tuning frequency of $230.55 \mathrm{GHz}$ in the upper sideband $\left(v_{\mathrm{lsr}}=59.1 \mathrm{~km} \mathrm{~s}^{-1}\right)$. Parts of the correlator were mis-functioning, narrowing down the usable bandwidth to approximately $1.6 \mathrm{GHz}$, the covered spectral range was 219.45 to $221.07 \mathrm{GHz}$ in the lower sideband (lsb) and 229.18 to $230.72 \mathrm{GHz}$ in the upper sideband (usb), respectively. The spectral resolution was $0.8125 \mathrm{kHz} /$ channel corresponding to $\sim 1.1 \mathrm{~km} \mathrm{~s}^{-1}$ velocity resolution. The weather was excellent with zenith opacities $\tau(225 \mathrm{GHz})$ between 0.1 and 0.07 measured by the National Radio Astronomy Observatory (NRAO) tipping radiometer operated by the Caltech Submillimeter Observatory (CSO). Passband and flux calibration were derived from Uranus observations, and the flux density scale is estimated to be accurate within $20 \%$. Phase and amplitude were calibrated with regularly interleaved observations of the quasar NRAO530 (11.8 ${ }^{\circ}$ from the source). We applied different weightings for the continuum and line data resulting in synthesized beams between $3.6^{\prime \prime} \times 2.4^{\prime \prime}$ and $3.9^{\prime \prime} \times 2.5^{\prime \prime}$. The rms noise of the line and continuum images is dominated by the side-lobes and the corresponding inadequate cleaning. The dirty beam with side-lobes at approximately a $40 \%$ level is shown in Fig. 1 . The $3 \sigma \mathrm{rms}$ of the $1.3 \mathrm{~mm}$ continuum image is $\sim 15 \mathrm{mJy}$. The varying rms of the different line images is discussed at the appropriate places in Sects. 3.2 and 3.3. The initial flagging and calibration was done with the IDL superset MIR originally developed for the Owens Valley Radio Observatory (Scoville et al. 1993) and adapted for the $\mathrm{SMA}^{2}$. The imaging and data analysis were conducted in MIRIAD (Sault et al. 1995).

\subsection{Short spacings from the IRAM $30 \mathrm{~m}$}

To complement the $\mathrm{CO}(2-1)$ observations with the missing short spacing information, we combined the SMA data with the

2 The MIR cookbook by Charlie Qi can be found at http: //cfa-www . harvard. edu/ cqi/mircook. html
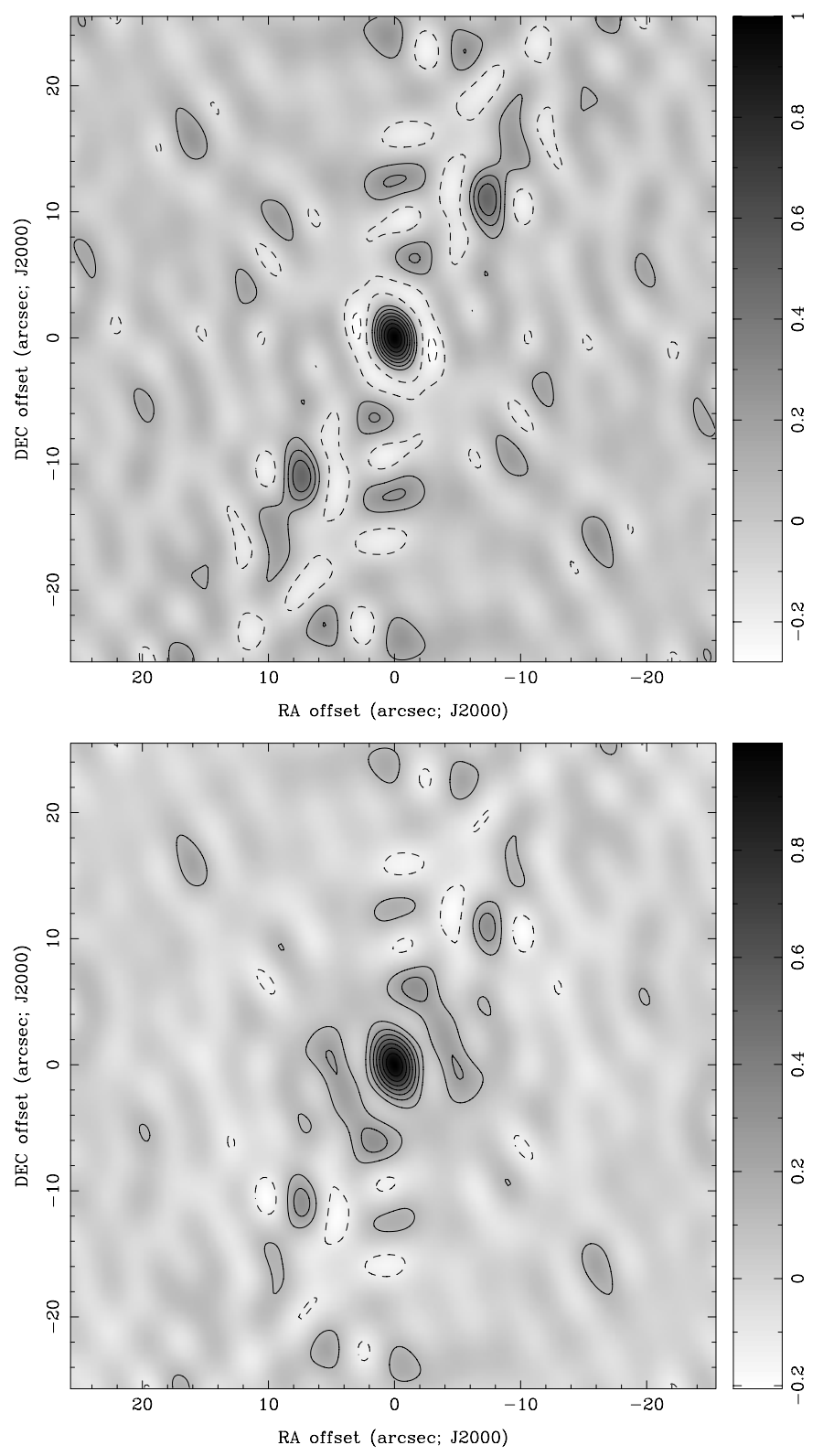

Fig. 1. The dirty beams of the SMA data alone (top panel) and the merged SMA+30 m data (bottom panel). The contour levels cover the range from \pm 1 in steps of \pm 0.125 .

previously published $\mathrm{CO}(2-1)$ map from the IRAM $30 \mathrm{~m}$ telescope (Beuther et al. 2002b). For observing details of the singledish observations we refer to the paper by Beuther et al. (2002b). The single-dish data were converted to visibilities within the MIRIAD package (using the task UVMODEL). The single-dish and interferometer data were then subsequently processed together. The weighting of the two datasets was chosen to recover large-scale emission but maintain at the same time the highspatial resolution to resolve small-scale structure. Convolving the merged dataset to the $11^{\prime \prime}$ beam of the $30 \mathrm{~m}$ data alone, the peak fluxes in the channel map are at approximately a $70 \%$ level of the single-dish measured fluxes. Measuring the integrated fluxes toward the same outflow regions for the merged dataset and the single-dish data alone, we even recover in the merged dataset $\sim 88 \%$ and $\sim 89 \%$ of the blue and red $\mathrm{CO}(2-1)$ singledish emission, respectively. The resulting dirty beam from the 


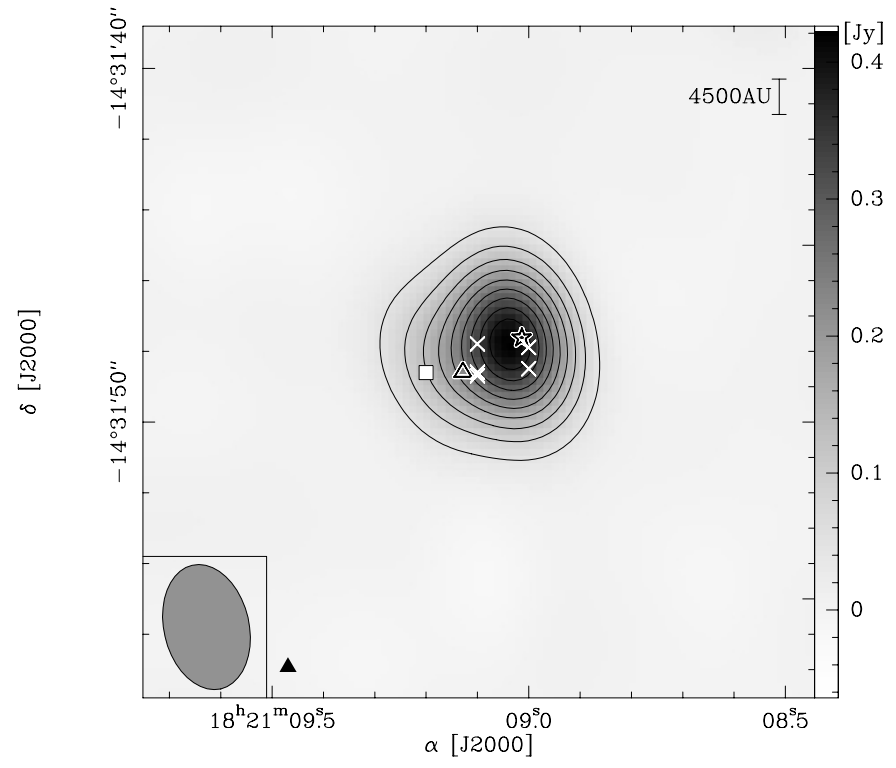

Fig. 2. $1.3 \mathrm{~mm}$ continuum image of IRAS $18182-1433$. The contours range from 10 to $90 \%$ (step 10\%) from the peak flux shown in Table 1. The synthesized beam $\left(3.6^{\prime \prime} \times 2.4^{\prime \prime}\right.$ PA $\left.13.9^{\circ}\right)$ is shown at the bottomleft. The star marks the $7 \mathrm{~mm}$ source and the open and filled triangles the $\mathrm{cm}$ sources with positive and negative spectral index, respectively (Zapata et al. 2005). The square and white crosses mark the positions from the $\mathrm{CH}_{3} \mathrm{OH}$ Class II and $\mathrm{H}_{2} \mathrm{O}$ masers, respectively (Walsh et al. 1998; Beuther et al. 2002c).

merged dataset is shown in Fig. 1 as well. The synthesized beam of the combined data is $4.2^{\prime \prime} \times 2.8^{\prime \prime}\left(\mathrm{PA} 15^{\circ}\right)$.

\section{Results}

\subsection{Millimeter continuum emission}

Figure 2 presents the synthesized $1.3 \mathrm{~mm}$ continuum image of the region produced by averaging the apparently line-free parts of the upper and lower sideband spectra presented in Fig. 3. We identify only one massive isolated source at the given resolution and sensitivity. The $7 \mathrm{~mm}$ continuum source from Zapata et al. (2005) is associated with the $1.3 \mathrm{~mm}$ peak position, whereas the $\mathrm{cm}$ source with positive spectral index - thus likely a thermal jet feature - is approximately $2^{\prime \prime}$ to the south-east (Zapata et al. 2005) and appears to be associated with the elongation of the $1.3 \mathrm{~mm}$ emission in this direction. The $\mathrm{CH}_{3} \mathrm{OH}$ Class II maser feature (Walsh et al. 1998) is probably rather associated with the $\mathrm{cm}$ jet feature than with the mm continuum peak. $\mathrm{H}_{2} \mathrm{O}$ maser emission shows various maser features distributed over the region (Beuther et al. 2002c), some of them appear to be associated with the $\mathrm{cm}$ feature whereas others are found closer to the $\mathrm{mm}$ continuum peak. The weak mid-infrared feature detected by De Buizer et al. (2005) may be more closely associated with the $\mathrm{cm}$ feature than with the mm peak. However, the given midinfrared astrometry $\left(\sim 1.5^{\prime \prime}\right)$ is not good enough to clearly associate that feature with one or the other peak, and we refrain from further interpretation of potential association between the midinfrared and $\mathrm{mm} / \mathrm{cm}$ continuum emission. Although the spatial resolution of the $1.3 \mathrm{~mm}$ continuum data is insufficient, the continuum extension in the direction of the $\mathrm{cm}$ jet feature indicates that the mm continuum emission is not only of protostellar nature but that there can be additional mm continuum emission due to an underlying jet (for other similar examples see Gueth et al. 2003 or Beuther et al. 2004b). Nevertheless, the majority of the

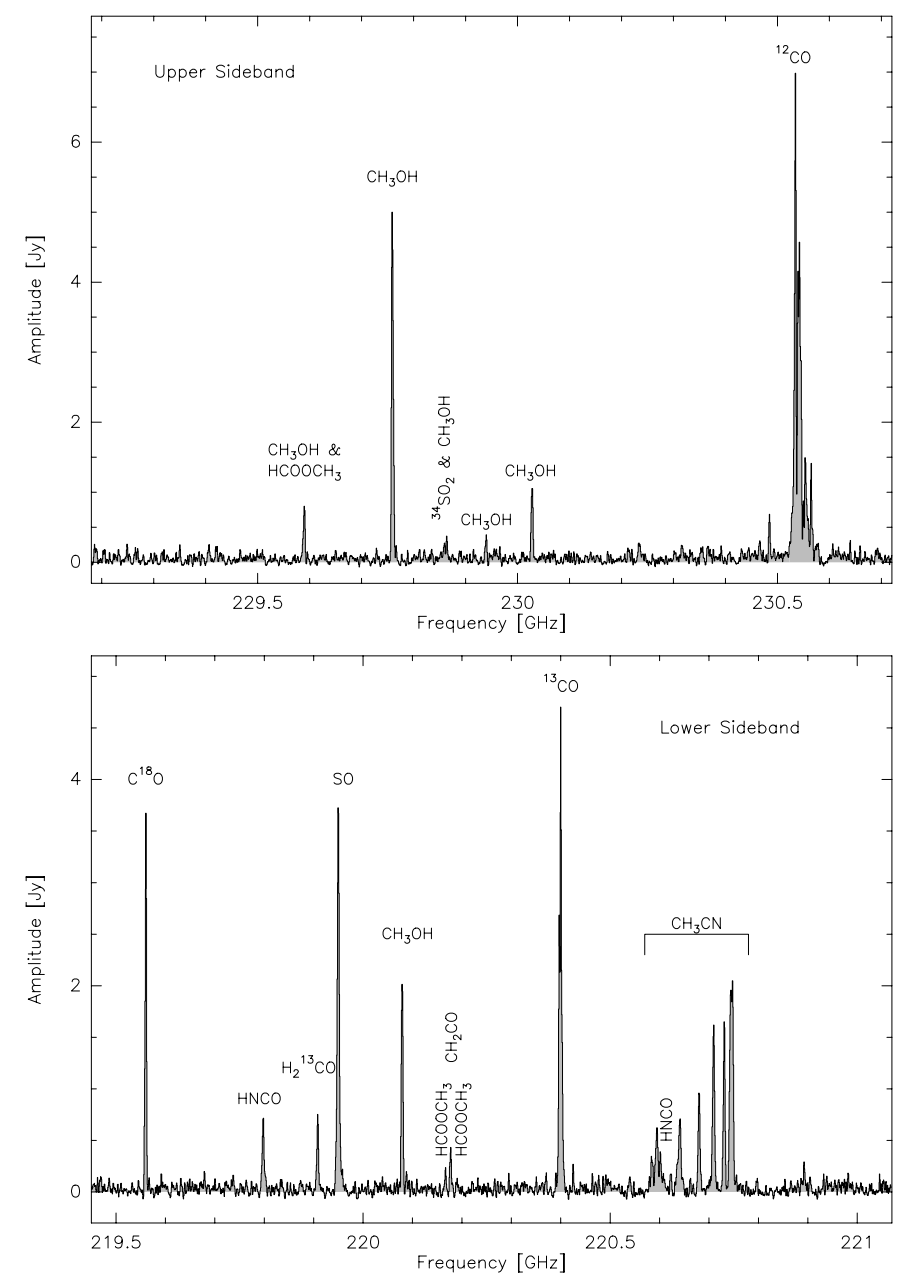

Fig. 3. Upper and lower sideband spectra vector-averaged over all baselines. The molecular lines are marked.

Table 1. Millimeter continuum data.

\begin{tabular}{lr}
\hline \hline$S_{\text {peak }}$ & $426 \mathrm{mJy} /$ beam \\
$S_{\text {int }}$ & $546 \mathrm{mJy}$ \\
Mass@43 K & $47.6 M_{\odot}$ \\
$N_{\mathrm{H}_{2}} @ 43 \mathrm{~K}$ & $5.7 \times 10^{23} \mathrm{~cm}^{-2}$ \\
Mass@ $150 \mathrm{~K}$ & $12.4 M_{\odot}$ \\
$N_{\mathrm{H}_{2}} @ 150 \mathrm{~K}$ & $1.5 \times 10^{23} \mathrm{~cm}^{-2}$ \\
\hline
\end{tabular}

mm continuum emission is due to cold dust from the protostellar object(s).

Compared with the single-dish flux at $1.2 \mathrm{~mm}$ wavelength of 3.9 Jy (Beuther et al. 2002a), the integrated interferometer flux of $\sim 546 \mathrm{mJy}$ is rather low, of the order $13 \%$ from the single-dish measurements. Since these interferometer observations are not sensitive to spatial scales $>25^{\prime \prime}$, we filter out a rather smoothly distributed large-scale halo, and only the compact core remains. Assuming optically thin dust emission, we can estimate the mass and column density of this central $1.3 \mathrm{~mm}$ dust and gas core following the equations outlined in Hildebrand (1983) and Beuther et al. (2002a). We calculate these parameters assuming two temperatures, $43 \mathrm{~K}$ as derived from two-components greybody fits (Sridharan et al. 2002), and $150 \mathrm{~K}$ consistent with the $\mathrm{CH}_{3} \mathrm{CN}\left(12_{k}-11_{k}\right)$ emission (see Sect. 3.5). Based on previous interferometric observations of similar sources from the original sample (IRAS 18089-1732 and IRAS 20293+3952, Beuther et al. 2004a,b), we use a dust opacity index $\beta$ of 1 , corresponding 
Table 2. Observed lines.

\begin{tabular}{|c|c|c|}
\hline $\begin{array}{l}v \\
{[\mathrm{GHz}]}\end{array}$ & $\overline{\text { line }}$ & $\begin{array}{r}E_{\text {lower }} \\
{[\mathrm{K}]}\end{array}$ \\
\hline \multicolumn{3}{|l|}{ LSB } \\
\hline 219.560 & $\mathrm{C}^{18} \mathrm{O}(2-1)$ & 5.3 \\
\hline 219.798 & $\operatorname{HNCO}\left(10_{0,10}-9_{0,9}\right)$ & 48 \\
\hline 219.909 & $\mathrm{H}_{2}^{13} \mathrm{CO}\left(3_{1,2}-2_{1,1}\right)$ & 22 \\
\hline 219.949 & $\mathrm{SO}\left(6_{5}-5_{4}\right)$ & 24 \\
\hline 220.079 & $\mathrm{CH}_{3} \mathrm{OH}\left(8_{0,8}-7_{1,6}\right)$ & 85 \\
\hline 220.167 & $\mathrm{HCOOCH}_{3}\left(17_{2,15}-16_{4,2}\right)$ & 93 \\
\hline 220.178 & $\mathrm{CH}_{2} \mathrm{CO}\left(1_{1,11}-10_{1,10}\right)$ & 66 \\
\hline 220.190 & $\mathrm{HCOOCH}_{3}\left(17_{4,13}-16_{4,12}\right)$ & 93 \\
\hline 220.399 & ${ }^{13} \mathrm{CO}(2-1)$ & 5.3 \\
\hline 220.585 & $\operatorname{HNCO}\left(10_{1,9}-9_{1,8}\right)$ & 91 \\
\hline 220.594 & $\mathrm{CH}_{3} \mathrm{CN}\left(12_{6}-11_{6}\right)$ & 315 \\
\hline 220.641 & $\mathrm{CH}_{3} \mathrm{CN}\left(12_{5}-11_{5}\right)$ & 237 \\
\hline 220.679 & $\mathrm{CH}_{3} \mathrm{CN}\left(12_{4}-11_{4}\right)$ & 173 \\
\hline 220.709 & $\mathrm{CH}_{3} \mathrm{CN}\left(12_{3}-11_{3}\right)$ & 123 \\
\hline 220.730 & $\mathrm{CH}_{3} \mathrm{CN}\left(12_{2}-11_{2}\right)$ & 87 \\
\hline 220.743 & $\mathrm{CH}_{3} \mathrm{CN}\left(12_{1}-11_{1}\right)$ & 65 \\
\hline 220.747 & $\mathrm{CH}_{3} \mathrm{CN}\left(12_{0}-11_{0}\right)$ & 58 \\
\hline \multicolumn{3}{|l|}{ USB } \\
\hline 229.589 & $\mathrm{CH}_{3} \mathrm{OH}\left(15_{4,11}-16_{3,13}\right) \mathrm{E}$ & 362 \\
\hline 229.590 & $\mathrm{HCOOCH}_{3}\left(19_{3,16}-18_{4,15}\right) \mathrm{E}$ & 106 \\
\hline 229.759 & $\mathrm{CH}_{3} \mathrm{OH}\left(8_{1,8}-7_{0,7}\right) \mathrm{E}$ & 77 \\
\hline 229.858 & ${ }^{34} \mathrm{SO}_{2}\left(4_{2,2}-3_{1,3}\right)$ & 7.7 \\
\hline 229.864 & $\mathrm{CH}_{3} \mathrm{OH}\left(19_{5,15}-20_{4,16}\right) \mathrm{A}+$ & 568 \\
\hline 229.939 & $\mathrm{CH}_{3} \mathrm{OH}\left(19_{5,14}-20_{4,17}\right) \mathrm{A}-$ & 568 \\
\hline 230.027 & $\mathrm{CH}_{3} \mathrm{OH}\left(3_{2,2}-4_{1,4}\right) \mathrm{E}$ & 28 \\
\hline 230.538 & ${ }^{12} \mathrm{CO}(2-1)$ & 5.5 \\
\hline
\end{tabular}

to a dust opacity per unit dust mass of $\kappa(1.3 \mathrm{~mm}) \sim 1.8 \mathrm{~cm}^{2} \mathrm{~g}^{-1}$. We note that $\beta=1.4$ corresponds to $\kappa(1.3 \mathrm{~mm}) \sim 0.9 \mathrm{~cm}^{2} \mathrm{~g}^{-1}$, as suggested by Ossenkopf \& Henning 1994, and using a larger $\kappa$ as we do results in lower mass estimates. Depending on the temperature, the central core mass is between 12.4 and $47.6 M_{\odot}$. The $\mathrm{H}_{2}$ column density varies between $1.5 \times 10^{23}$ and $5.7 \times 10^{23} \mathrm{~cm}^{-2}$ (Table 1), corresponding to visual extinctions of a few hundred $\left(A_{v}=N_{\mathrm{H}_{2}} / 0.94 \times 10^{21}\right.$, Frerking et al. 1982). At a temperature of $43 \mathrm{~K}$, the $3 \sigma \mathrm{rms}$ of $15 \mathrm{mJy}$ corresponds to a mass sensitivity of $\sim 1.3 M_{\odot}$.

We tried to derive radial intensity profiles, but since the core is only marginally resolved and the data suffer strongly from the missing short spacings, the resulting intensity distribution is unreasonably steep prohibiting a further analysis in this direction.

\subsection{Spectral line emission}

Figure 3 presents the observed spectral bandpass around 220 and $230 \mathrm{GHz}$ in the lsb and usb, respectively. Altogether we detect 25 lines from 8 species $\left({ }^{12} \mathrm{CO}, \mathrm{HNCO}, \mathrm{H}_{2}^{13} \mathrm{CO}, \mathrm{SO},{ }^{34} \mathrm{SO}_{2}\right.$, $\mathrm{CH}_{3} \mathrm{OH}, \mathrm{HCOOCH}_{3}, \mathrm{CH}_{3} \mathrm{CN}$ ) and 2 additional $\mathrm{CO}$ isotopologues $\left({ }^{13} \mathrm{CO}\right.$ and $\left.\mathrm{C}^{18} \mathrm{O}\right)$ with lower energy levels $E_{\text {lower }}$ between 5 and $568 \mathrm{~K}$ (Table 2). The broad range of energy levels shows the power of broad spectral bandpasses which allow to study very different excitation regimes simultaneously.

Figure 4 presents integrated line images of all species and isotopologues (except ${ }^{34} \mathrm{SO}_{2}$ which is blended with $\mathrm{CH}_{3} \mathrm{OH}$ and too weak for imaging). For ${ }^{12} \mathrm{CO}$ and ${ }^{13} \mathrm{CO}$ we are showing the blue and red line wing emission identifying a bipolar outflow centered on the mm continuum peak. While the blue ${ }^{12} \mathrm{CO}$ emission is confined to a small region south-east of the mm-peak, the red ${ }^{12} \mathrm{CO}$ emission shows cone-like emission toward the northwest. The outflow will be discussed in detail in Sect. 3.3. All other spectral lines show compact emission close to the $\mathrm{mm}$ emission peak. Interestingly none of these species peak exactly toward the mm continuum, but all peak positions are shifted a little bit to the south-east (of the order $1-2^{\prime \prime}$ ), largely coinciding with the $\mathrm{cm}$ emission peak attributed to the thermal jet (Zapata et al. 2005). Although the shift in the spectral line maps is smaller than the angular resolution of the synthesized beam, we consider it a real observational feature because (a) the line and continuum images are extracted from the same dataset, and (b) by fitting peak positions one can achieve a significant higher angular resolution than the actual beam size $H P B W$ depending on the signal to noise ration $S / N$ (down to $0.5 \times H P B W /(S / N)$, Reid et al. 1988). A close inspection of the higher density tracing ${ }^{13} \mathrm{CO}$ blue and red outflow emission shows that both line wings shows strong emission toward the south-east of the mm peak as well, and are thus also associated with the $\mathrm{cm}$ jet. A spatial offset between most molecular species - particularly high-density tracers like $\mathrm{CH}_{3} \mathrm{CN}$ or $\mathrm{HCOOCH}_{3}$ - and the mm continuum emission is surprising, and various potential origins will be discussed in Sect. 4.

\subsection{The molecular outflows}

Figure 5 shows the SMA-only ${ }^{12} \mathrm{CO},{ }^{13} \mathrm{CO}$ and $\mathrm{C}^{18} \mathrm{O}$ spectra vector-averaged over all baselines. The ${ }^{12} \mathrm{CO}$ spectrum shows a dip around the systemic velocity $v_{\text {lsr }}$ of $59.1 \mathrm{~km} \mathrm{~s}^{-1}$, which is not real absorption but caused by the missing short-spacing information in the interferometer data. The interferometric spatial filtering affects the most abundant isotopologue ${ }^{12} \mathrm{CO}$ strongest because it is optically thick at the $v_{\text {lsr }}$, tracing the extended cloud envelope. In contrast to that, the less abundant isotopologues ${ }^{13} \mathrm{CO}$ and $\mathrm{C}^{18} \mathrm{O}$ have lower opacities, they are tracing more compact structures and hence do not show the pronounced dip at the $v_{\text {lsr }}$. Nevertheless, already these vector-averaged spectra in the uv-domain show ${ }^{12} \mathrm{CO}$ high-velocity line wings due to the molecular outflow between 30 and $100 \mathrm{~km} \mathrm{~s}^{-1}$. Although weaker and spanning a narrower velocity range, the line-wing emission is also found in the ${ }^{13} \mathrm{CO}$ data but barely discernable in the rare isotopologue $\mathrm{C}^{18} \mathrm{O}$.

Since we have the corresponding ${ }^{12} \mathrm{CO}(2-1)$ single-dish observations from the IRAM $30 \mathrm{~m}$ telescope (Beuther et al. 2002b, and Fig. 8 top-left panel), we were able to combine interferometer and single-dish data and thus recover the missing short spacings. The IRAM $30 \mathrm{~m}$ data were less sensitive than the new SMA observations detecting high-velocity emission only approximately between 50 and $70 \mathrm{~km} \mathrm{~s}^{-1}$. Therefore, we confined the SMA data to the range between 47.5 and $72.5 \mathrm{~km} \mathrm{~s}^{-1}$ to combine both datasets. Figure 6 presents a ${ }^{12} \mathrm{CO}(2-1)$ channel map showing the merged SMA $+30 \mathrm{~m}$ data between 50 and $70 \mathrm{~km} \mathrm{~s}^{-1}$ and the SMA only data at higher velocities relative to the $v_{\mathrm{lsr}}$. Figure 8 shows the resulting outflow images covering all spatial scales from the single-dish to the interferometer observations.

The interferometer data alone exhibit $\mathrm{CO}$ emission approximately between 30 and $100 \mathrm{~km} \mathrm{~s}^{-1}$ (Figs. 6 and 7). We tried to image the $24 \mathrm{~km} \mathrm{~s}^{-1}$ feature (Fig. 5) but could not identify any clear signature associated with IRAS 18182-1433. Therefore, we conclude that this spectral feature has to be associated with an unrelated fore- or background cloud. The red-shifted feature in the SMA-only CO outflow image in Fig. 8 (top-middle panel) at Declination $\sim-11^{\prime \prime}$ and RA between approximately $-5^{\prime \prime}$ and $-20^{\prime \prime}$ is an artifact of the image deconvolution and the strong side-lobes caused by the insufficient uv-coverage and missing short-spacings of the SMA data alone. After adding in the short spacing information from the IRAM $30 \mathrm{~m}$ observations, 


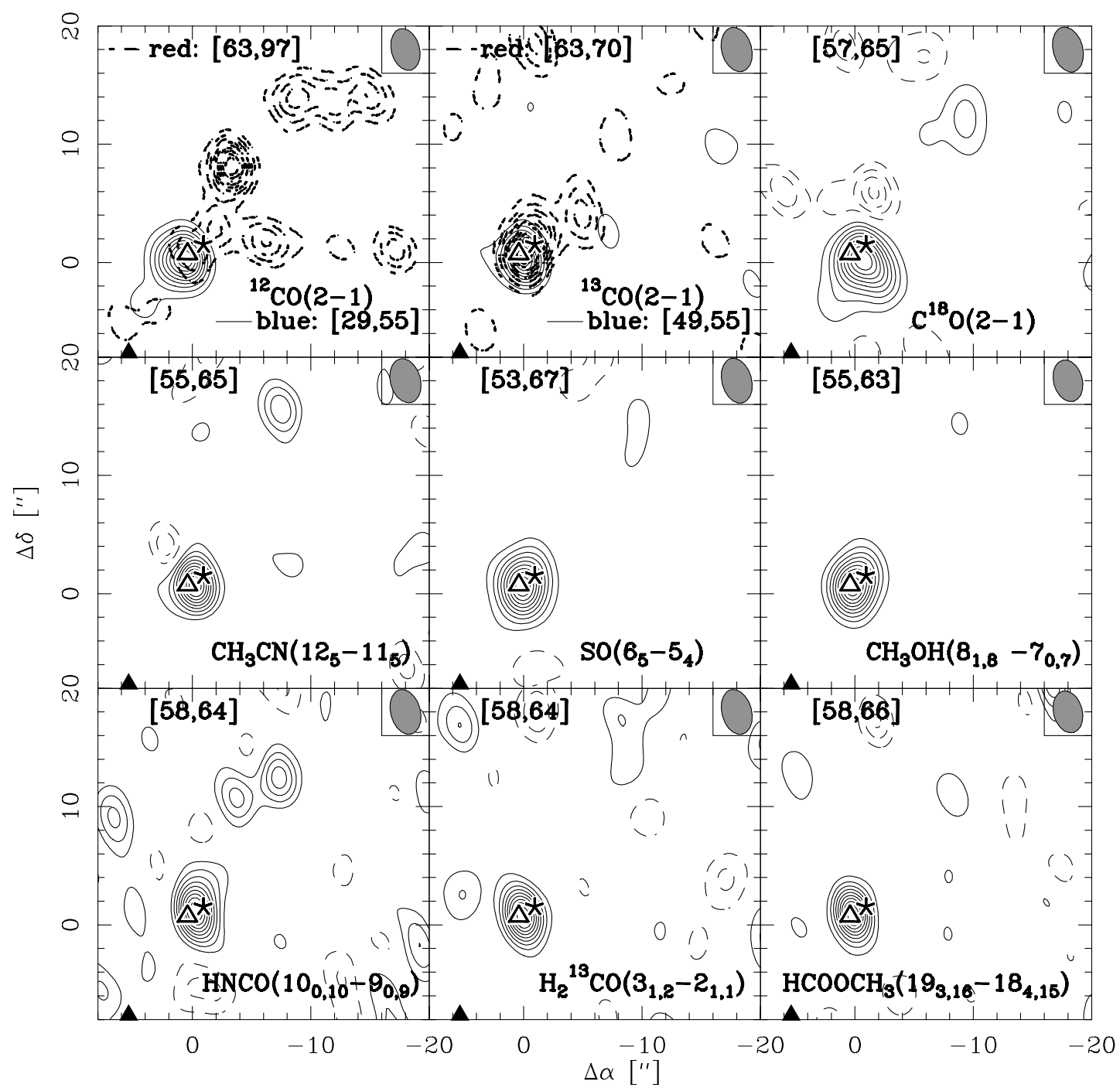

Fig. 4. Integrated images of the observed molecular species. The full and dashed contours present the integrated emission as labeled in ${ }^{12} \mathrm{CO}$ and ${ }^{13} \mathrm{CO}$ image. The full and dashed contours in the remaining panels present the positive and negative (due to the side-lobe emission and inadequate cleaning) integrated emission as labeled in the panels. The contour levels range from 10 to $90 \%$ (step 10\%) of the peak emission reported in Table 3. There we also list the $1 \sigma \mathrm{rms}$ of each integrated image. The star marks the $1.3 \mathrm{~mm}$ continuum peak position from Fig. 2, the open and full triangle mark the $\mathrm{cm}$ emission sources with positive and negative spectral index, respectively (Zapata et al. 2005). The synthesized beams are shown at the top-right of each panel.

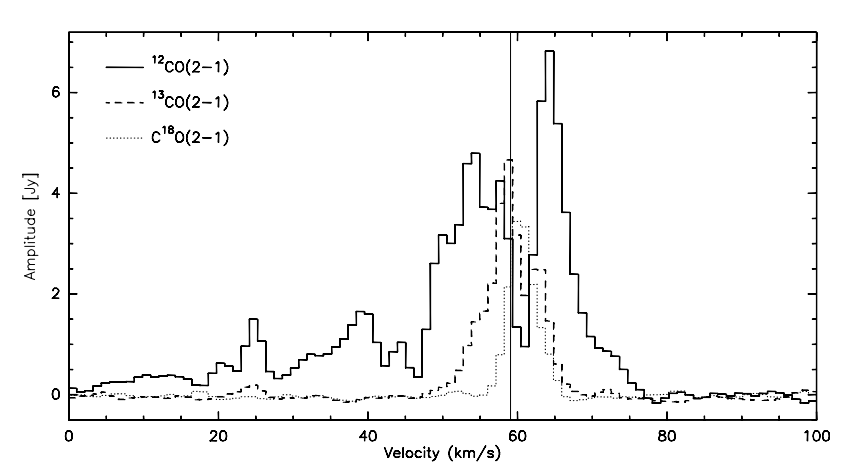

Fig. 5. SMA-only ${ }^{12} \mathrm{CO},{ }^{13} \mathrm{CO}$ and $\mathrm{C}^{18} \mathrm{O}(2-1)$ spectra vector-averaged over all baselines. The spectral resolution is the nominal resolution of $1.1 \mathrm{~km} \mathrm{~s}^{-1}$. The $v_{\mathrm{lsr}}$ at $59.1 \mathrm{~km} \mathrm{~s}^{-1}$ is marked by a vertical line.

this feature is largely gone, whereas the southern edge of the red-shifted CO cone at Declination $0^{\prime \prime}$ is recovered much better (Figs. 6 and 8).
Table 3. Line image parameters to Fig. 4.

\begin{tabular}{lrr}
\hline \hline line & $\begin{array}{r}1 \sigma \\
{\left[\frac{\mathrm{mJy}}{\text { beam }} \frac{\mathrm{km}}{\mathrm{s}}\right]}\end{array}$ & $\begin{array}{r}S_{\text {peak }} \\
{\left[\frac{\mathrm{Jy}}{\text { beam }} \frac{\mathrm{km}}{\mathrm{s}}\right]}\end{array}$ \\
\hline${ }^{12} \mathrm{CO}(2-1)$ blue & 1040 & 37.7 \\
${ }^{12} \mathrm{CO}(2-1)$ red & 1088 & 29.2 \\
${ }^{13} \mathrm{CO}(2-1)$ blue & 192 & 4.2 \\
${ }^{13} \mathrm{CO}(2-1)$ red & 224 & 5.1 \\
$\mathrm{C}^{18} \mathrm{O}(2-1)$ & 440 & 13.3 \\
$\mathrm{CH}_{3} \mathrm{CN}\left(12_{5}-11_{5}\right)$ & 200 & 4.6 \\
$\mathrm{SO}\left(6_{5}-5_{4}\right)$ & 630 & 22.0 \\
$\mathrm{CH} \mathrm{H}_{3} \mathrm{OH}\left(8_{1,8}-7_{0,7}\right) \mathrm{E}$ & 480 & 24.9 \\
$\mathrm{HNCO}\left(10_{0,10}-9_{0,9}\right)$ & 240 & 2.4 \\
$\mathrm{H}_{2}^{13} \mathrm{CO}\left(3_{1,2}-2_{1,1}\right)$ & 168 & 2.8 \\
$\mathrm{HCOOCH}{ }_{3}\left(19_{3,16}-18_{4,15}\right) \mathrm{E}$ & 224 & 4.3 \\
\hline
\end{tabular}

We find red and blue outflow emission between 30 and $70 \mathrm{~km} \mathrm{~s}^{-1}$ south-east of the mm peak, whereas toward the north-west only red CO emission is detected. The north-western 


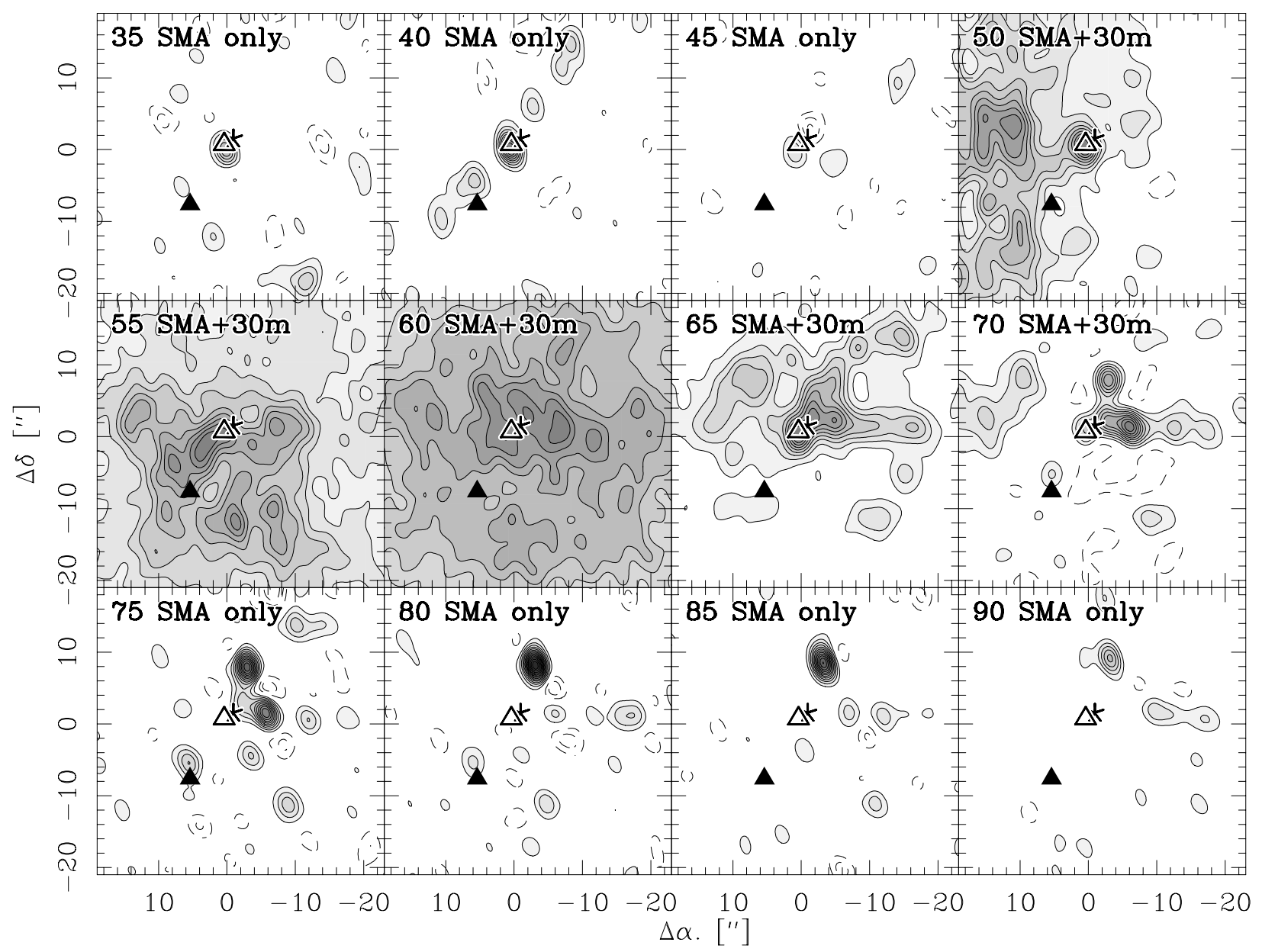

Fig. 6. ${ }^{12} \mathrm{CO}(2-1)$ channel map with $5 \mathrm{~km} \mathrm{~s}^{-1}$ resolution. The channels between 35 to $45 \mathrm{~km} \mathrm{~s}^{-1}$ and between 75 to $90 \mathrm{~km} \mathrm{~s}^{-1}$ are produced from SMA only data, whereas the channels inbetween are from the merged SMA+30M dataset (labeled in each panel with the corresponding central velocity). For the SMA only data, we plot the contour levels from $\pm 0.5 \mathrm{Jy}_{\text {beam }}^{-1} \mathrm{~km} \mathrm{~s}^{-1}$ in $\pm 0.5 \mathrm{Jy} \mathrm{beam}^{-1} \mathrm{~km} \mathrm{~s}^{-1}$ steps. The $1 \sigma$ rms of the weak emission channel at $90 \mathrm{~km} \mathrm{~s}^{-1}$ is $0.15 \mathrm{Jy} \mathrm{beam}^{-1} \mathrm{~km} \mathrm{~s}^{-1}$. The SMA+30 m data are contoured differently in each panel to account for the dynamic range differences. The start- and step-values are the same in units of Jy beam ${ }^{-1} \mathrm{~km} \mathrm{~s}^{-1} ; 50 \mathrm{~km} \mathrm{~s}^{-1}: \pm 1.6 ; 55 \mathrm{~km} \mathrm{~s}^{-1}: \pm 4.4 ; 60 \mathrm{~km} \mathrm{~s}^{-1}: \pm 6.6$; $65 \mathrm{~km} \mathrm{~s}^{-1}: \pm 3.4 ; 70 \mathrm{~km} \mathrm{~s}^{-1}: \pm 1.0$. The crosses mark the position of the $\mathrm{mm}$ continuum peak, and the open and full triangles mark the positions of the $\mathrm{cm}$ sources with positive and negative spectral index (Zapata et al. 2005).

emission exhibits a clear cone-like morphology, whereas the south-eastern emission appears jet-like with the compact component being associated with the thermal jet feature observed in $\mathrm{cm}$ emission (Zapata et al. 2005). Integrating over slightly different velocity regimes for the blue wing, the observed structures do vary a bit: employing the originally from the singledish data alone identified blue range from 53 to $57 \mathrm{~km} \mathrm{~s}^{-1}$, the south-eastern jet-feature dominates the blue wing of this outflow. However, integrating a little bit broader regime from 48 to $58 \mathrm{~km} \mathrm{~s}^{-1}$, one can tentatively identify associated structures at offset $\left(10^{\prime \prime} /-13^{\prime \prime}\right)$ and $\left(12^{\prime \prime} / 3^{\prime \prime}\right)$ which could be part of a blue counter-cone. This potential blue cone-like morphology is less prominent than for the red north-western wing, and with this dataset we cannot unambiguously decide whether the blue outflow wing only shows jet-like emission or whether it actually features a cone-like morphology.

The single-dish data alone indicated an outflow oriented roughly in north-south direction whereas the interferometer data suggest an orientation approximately in north-west south-east direction. The combined data now show that we are likely dealing with a system of multiple outflows: the one outflow (a) is the north-west south-east outflow revealed in detail by the SMA observations. However, most of the single-dish flux at blue-shifted

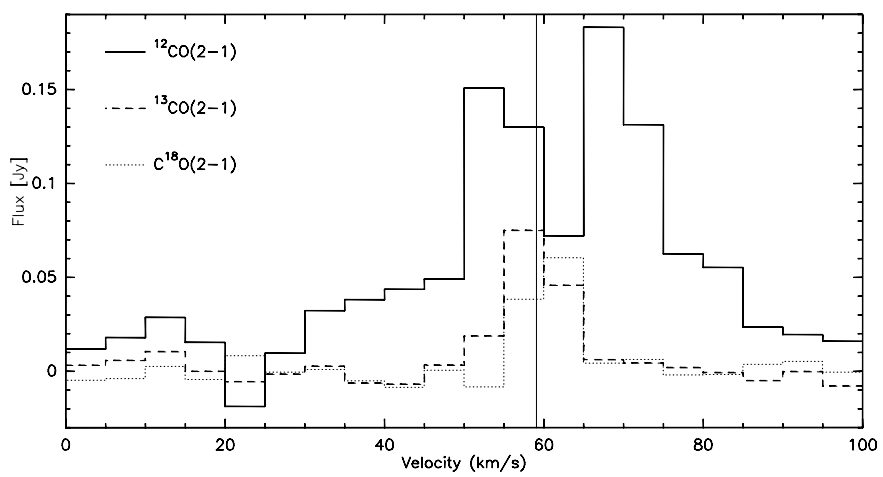

Fig. 7. SMA-only ${ }^{12} \mathrm{CO},{ }^{13} \mathrm{CO}$ and $\mathrm{C}^{18} \mathrm{O}(2-1)$ spectra extracted from the data cube after imaging with a spectral resolution of $5 \mathrm{~km} \mathrm{~s}^{-1}$. The spectra are averaged spatially over the whole region covered by the $\mathrm{CO}$ outflow (see top-left panel in Fig. 4). The $v_{\mathrm{lsr}}$ at $59.1 \mathrm{~km} \mathrm{~s}^{-1}$ is marked by a vertical line.

velocities is to the south of the mm continuum peak, and Fig. 8 shows additional red-shifted emission toward the north-east of it. One possibility would be that the whole blue/red outflow structure is due to one inhomogeneous wide-angle outflow with its 


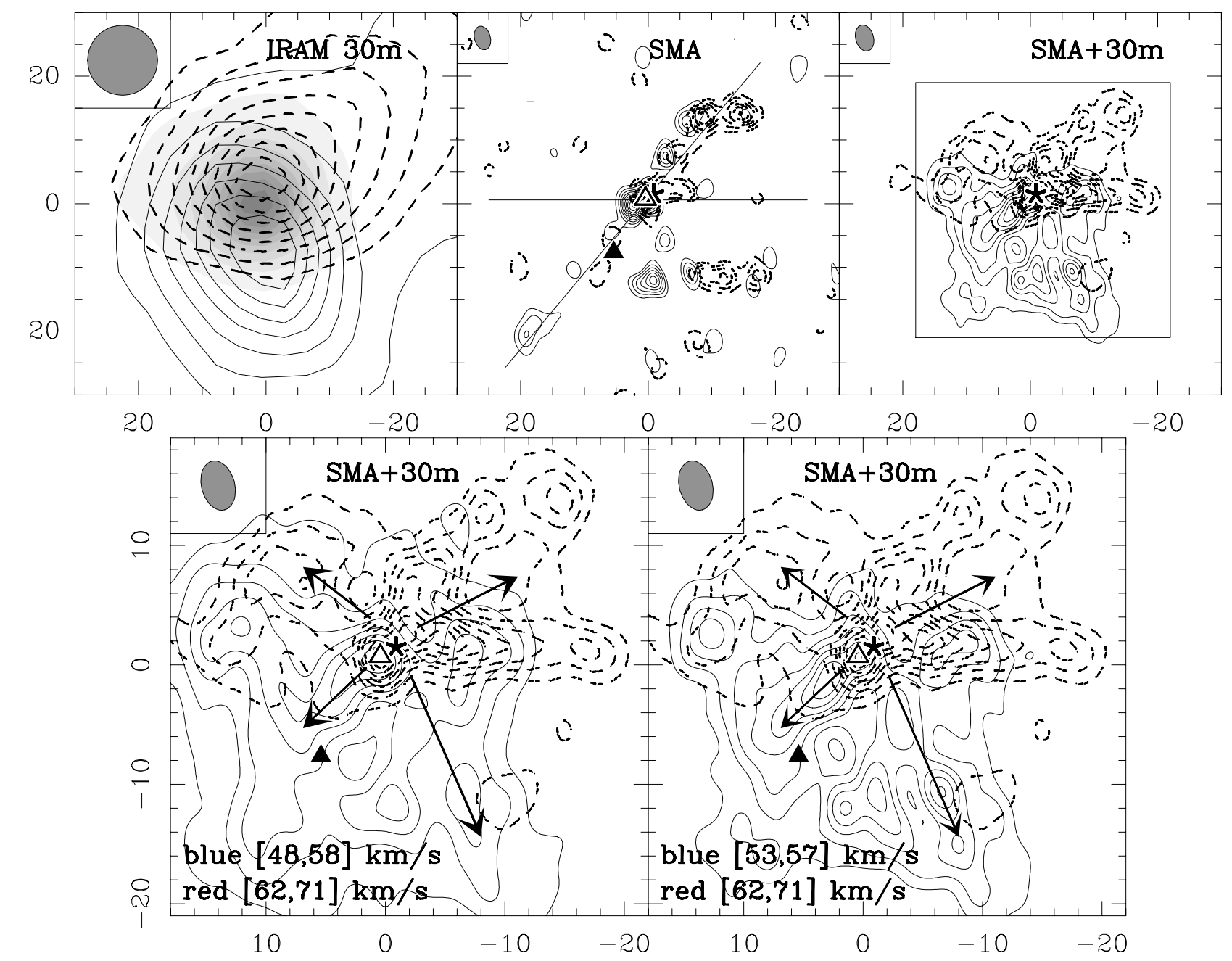

Fig. 8. ${ }^{12} \mathrm{CO}(2-1)$ outflow images observed with the IRAM $30 \mathrm{~m}$ telescope (Beuther et al. 2002b) and the SMA. The top-left panel shows the $30 \mathrm{~m}$ data only, the top-middle presents the SMA data only. The top-right and the bottom panels then show the combined SMA+30 m data. The velocity-integration regimes for the top-row panels and the bottom-right panel are: blue (full contours) $[53,57] \mathrm{km} \mathrm{s}^{-1}$ and red (dashed contours) $[62,71] \mathrm{km} \mathrm{s}^{-1}$. The bottom-left panel has a slightly broader blue velocity range from $[48,58] \mathrm{km} \mathrm{s}^{-1}$. The grey-scale in the top-left panel presents the $1.2 \mathrm{~mm}$ continuum emission from the IRAM $30 \mathrm{~m}$ (Beuther et al. 2002a). The stars in the other panels mark the mm continuum peak from the SMA observations. The open and full triangles in the top-middle and bottom panels mark the positions of the $\mathrm{cm}$ emission peaks with positive and negative spectral index, respectively (Zapata et al. 2005). The (synthesized) beams are shown at the top-left of each panel, and the axes are offsets in arcseconds from the phase center. The lines in the top-middle panel show the pv-cuts presented in Fig. 10, and the arrows in the bottom panel mark the approximate directions of the outflows discussed in the main text. The contour levels are listed in Table 4.

Table 4. Line image parameters to Fig. 8.

\begin{tabular}{lr}
\hline \hline & $\begin{array}{r}\text { Contours } \\
{\left[\mathrm{Jy} \mathrm{beam}^{-1} \mathrm{~km} \mathrm{~s}^{-1}\right]}\end{array}$ \\
\hline $30 \mathrm{~m}$ blue & $66(22) 198$ \\
$30 \mathrm{~m}$ red & $41(21) 185$ \\
SMA blue & $1.36(1.36) 12.24$ \\
SMA red & $2.07(2.07) 18.63$ \\
SMA+30 m blue & $10.92(3.60) 32.76$ \\
SMA+30 m red & $8.82(4.41) 39.69$ \\
\hline
\end{tabular}

symmetry axis along the jet direction. The features observed with the SMA would then potentially trace the denser parts of the molecular outflow. In contrast to this picture, the cone-like red-shifted emission as well as the jet-like blue-shifted features are strongly suggestive of at least one more homogeneous outflow in north-west south-east direction as discussed above. To account for the additional blue-shifted emission in the south and the red-shifted emission toward the north-east, we interpret these features as due to a second outflow (b) emanating from the same mm continuum core. The two outflows are inclined by approximately $90^{\circ}$ to each other (projected on the plane of the sky). The corresponding blue and red outflow lobes are for both outflows not exactly in opposite directions (as sketched in Fig. 8), but appear to be bend a little bit. Since we expect that the two outflows are interacting with each other, such an observed bending is no surprise but can be attributed to overlapping contributions from all outflows. In summary, the merged $\mathrm{CO}(2-1)$ dataset indicate a quadrupolar outflow system emanating from the main $\mathrm{mm}$ continuum source in IRAS 18182-1433.

To increase the complexity of the outflow system from IRAS 18182-1433, Fig. 9 presents an overlay of the $\mathrm{CO}(2-1)$ data from the $\mathrm{SMA}+30 \mathrm{~m}$ observations with VLA $\mathrm{SiO}(1-0)$ observations integrated over the velocity range from 40 to $90 \mathrm{~km} \mathrm{~s}^{-1}$ (kindly provided by Yuan Chen et al., in prep.). The south-eastern $\mathrm{SiO}$ feature is consistent with the blue jet-like $\mathrm{CO}$ emission, whereas the $\mathrm{SiO}$ emission north of the $\mathrm{mm}$ continuum peak shows a different morphology compared 


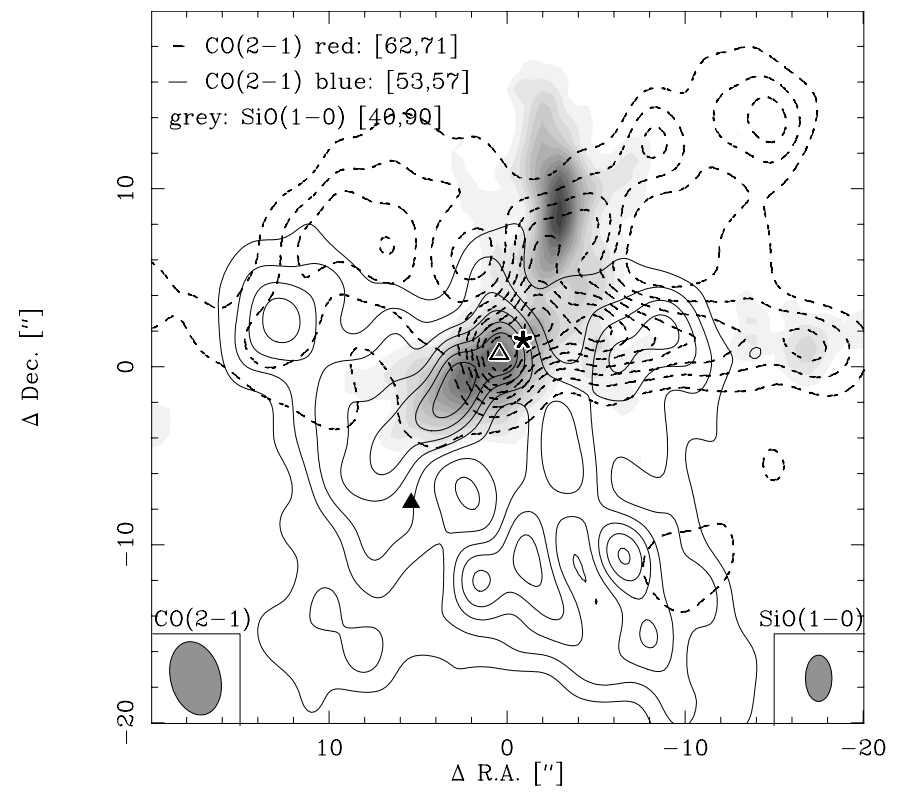

Fig. 9. The thin full and dashed contours show the same blue and red $\mathrm{CO}(2-1)$ observations merged from the SMA and IRAM $30 \mathrm{~m}$ data as presented in Fig. 8. The grey-scale shows the VLA SiO(1-0) emission integrated from 40 to $90 \mathrm{~km} \mathrm{~s}^{-1}$ (kindly provided by Yuan Chen et al., in prep.). The contour levels of the $\mathrm{CO}$ images are presented in Table 4, and the SiO data are contoured from 18 to $342 \mathrm{Jy} \mathrm{beam}^{-1} \mathrm{~km} \mathrm{~s}^{-1}$ (step $27 \mathrm{Jy} \mathrm{beam}^{-1} \mathrm{~km} \mathrm{~s}^{-1}$ ). The $\mathrm{CO}$ and $\mathrm{SiO}$ synthesized beams are shown at the bottom-left and bottom-right, respectively. The cross marks the position of the mm continuum peak, and the open and full triangles mark the positions of the $\mathrm{cm}$ sources with positive and negative spectral index, respectively (Zapata et al. 2005).

to the red $\mathrm{CO}$ cone-like outflow. The strong northern $\mathrm{SiO}$ peak position appears to be correlated with the northern edge of the $\mathrm{CO}$ cone in that direction. However, the rest of the $\mathrm{SiO}$ emission continues even further north, unrelated to the $\mathrm{CO}$ outflow. We detect additional weaker $\mathrm{SiO}$ emission at an offset $\left(-16^{\prime \prime} / 1^{\prime \prime}\right)$ spatially associated with the western end of the $\mathrm{CO}$ cone. The additional $\mathrm{SiO}$ peak at $\left(-16^{\prime \prime} / 1^{\prime \prime}\right)$ and the northern peak correlated with the northern $\mathrm{CO}$ cavity wall allow the interpretation that the $\mathrm{SiO}$ emission may be due to the same outflow observed in $\mathrm{CO}$, just more strongly excited in the cavity walls. However, the $\mathrm{SiO}$ extension further north is suggestive of an additional third outflow so far not detected in the $\mathrm{CO}$ emission.

Figure 10 presents position-velocity ( $\mathrm{pv}$ ) diagrams of the molecular gas along the directions of the two cavity walls of the north-western outflow cone. Both diagrams show that the highvelocity gas on the blue-shifted south-eastern side of the outflow remains very close to the outflow center whereas the red-shifted north-western side resembles more the typical Hubble-law with increasing velocity at further distances from the outflow center (e.g., Arce et al. 2006). This very different blue/red outflow pvcharacteristics are likely due to the fact that the blue CO outflow emission apparently traces predominantly the jet-like component also visible in $\mathrm{cm}$ and $\mathrm{SiO}$ emission, whereas the red $\mathrm{CO}$ emission is found mainly toward the cavity-like walls of the northwestern outflow component.

The combined $\mathrm{CO}(2-1)$ data can also be used to estimate the contributions of the two outflows emanating from IRAS $18182-1433$. To calculate the outflow mass $M\left(M_{\mathrm{blue}}\right.$, $\left.M_{\text {red }}, M_{\text {tot }}\right)$ the momentum $p$ and the energy $E$, we follow the approach outlined in Cabrit \& Bertout (1990) and already employed for the single-dish data alone by Beuther et al. (2002b).
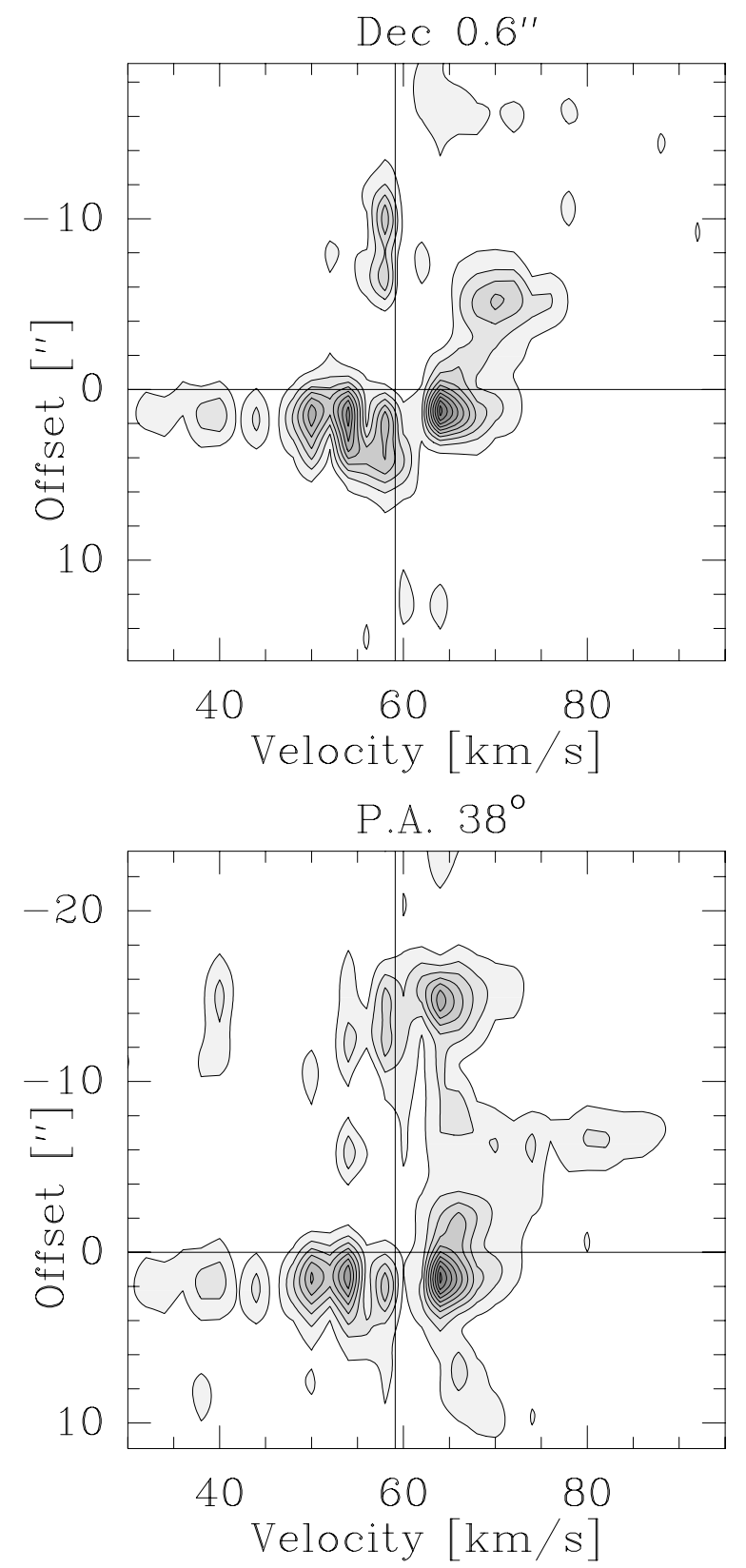

Fig. 10. Position-velocity diagrams for the ${ }^{12} \mathrm{CO}(2-1)$ SMA only outflow observations with a velocity resolution of $2 \mathrm{~km} \mathrm{~s}^{-1}$. The top panel is a pv-plot in RA at a Declination offset $+0.6^{\prime \prime}$ (corresponding to the strongest features in the west). The bottom panel presents a pv-diagram in north-west south-eastern direction with a PA of $38^{\circ}$ from north (the cuts are marked in Fig. 8, top-middle panel). The contour levels are from 5 to $95 \%$ from the peak emission ( $4.8 \mathrm{Jy} \mathrm{beam}^{-1}$ in both panels). The $v_{\mathrm{lsr}}$ at $59.1 \mathrm{~km} \mathrm{~s}^{-1}$ and the central positions are marked by vertical and horizontal lines.

In the following section (Sect. 3.4), we will discuss in more detail the varying $\mathrm{CO}$ line opacities, however, for the total parameters $M / p / E$ assuming an average ${ }^{12} \mathrm{CO} /{ }^{13} \mathrm{CO}(2-1)$ line ratio of 10 to calculate the opacity-corrected column densities of the blue- and red-shifted outflow emission is a reasonably approach (Choi et al. 1993; Levreault 1988; Beuther et al. $2002 b$ ). Furthermore, we use an $\mathrm{H}_{2} /{ }^{13} \mathrm{CO}$ ratio of $89 \times 10^{4}$ (Cabrit $\&$ Bertout 1992), an average temperature within the outflow of $30 \mathrm{~K}$, and the velocity ranges of $[53,57]$ and $[62,71] \mathrm{km} \mathrm{s}^{-1}$ for the integrated blue and red outflow wings, respectively. The derived masses, momenta and energies are shown in Table 5. 
Table 5. Outflow parameter.

\begin{tabular}{lrrrr}
\hline \hline & $30 \mathrm{~m}$ & SMA/30 m & SMA/30 m(a) & SMA/30 m(b) \\
\hline$M_{\text {blue }}\left[M_{\odot}\right]$ & 19.3 & 10.8 & 2.8 & 5.8 \\
$M_{\text {red }}\left[M_{\odot}\right]$ & 10.8 & 7.1 & 4.5 & 2.1 \\
$M_{\text {tot }}\left[M_{\odot}\right]$ & 30.1 & 17.9 & 7.3 & 7.9 \\
$p\left[M_{\odot} \mathrm{km} \mathrm{s}^{-1}\right]$ & 246 & 151 & 71 & 60 \\
$E\left[10^{45} \mathrm{erg}\right]$ & 23.0 & 14 & 8 & 5 \\
\hline
\end{tabular}

Although we recover approximately $88 \%$ of the red and blue wing emission when integrating over exactly the same region (see Sect. 2.2), the derived parameters from the merged dataset are a little bit lower because the area used to derive the outflow parameters from the single-dish data alone is larger than for the merged dataset (see Beuther et al. 2002b and Fig. 8). Interestingly, both outflows contribute approximately the same mass, momentum and energy to the total budget of the whole quadrupolar outflow system.

\subsection{Outflow line opacities}

The simultaneous observations of the ${ }^{12} \mathrm{CO}$ line and their isotopologues ${ }^{13} \mathrm{CO}$ and $\mathrm{C}^{18} \mathrm{O}$ (Figs. 5 and 7) should in principle allow to study the line wing ratios and the corresponding $\mathrm{CO}$ line wing opacities with changing velocity. The ${ }^{12} \mathrm{CO} /{ }^{13} \mathrm{CO}$ study by Choi et al. (1993) indicated that the ${ }^{12} \mathrm{CO}$ line opacities likely decrease with increasing velocity with respect to the $v_{\mathrm{lsr}}$ but the data were not sufficient to test this suggestion in more detail. Our new data toward IRAS 18182-1433 have the advantage that the simultaneous observation of the three lines minimizes the calibration uncertainties. However, the disadvantage is that the spatial filtering effect of an interferometer can vary for the different isotopologues. This is due to a number of reasons, two of them are: (1) varying line opacities between the isotopologues and thus different spatial structures traced by each of them. (2) The synthesized maps are affected by the side-lobes of the strongest emission features which causes different distortion of the weaker emission in various isotopologues. Since we have no corresponding single-dish ${ }^{13} \mathrm{CO}(2-1)$ and $\mathrm{C}^{18} \mathrm{O}(2-1)$ observations, it is hard to quantify this effect exactly for all three lines, but Beuther et al. (2005a) have shown for SMA observations of Orion-KL that this effect can significantly change the observed line ratios. Therefore, we stress that the absolute line ratios based on the SMA-data alone are not reliable. However, even the relative changes in the ${ }^{12} \mathrm{CO}(2-1) /{ }^{13} \mathrm{CO}(2-1)$ line ratio is interesting and allows conclusions about the changing line-wing opacities. Figure 11 presents the ${ }^{12} \mathrm{CO}(2-1) /{ }^{13} \mathrm{CO}(2-1)$ line ratio averaged over the whole region of the molecular outflow. We clearly see the trend of increasing line ratio with increasing velocity. While the ${ }^{12} \mathrm{CO}(2-1) /{ }^{13} \mathrm{CO}(2-1)$ line ratio is low close to the $v_{\text {lsr }}$, it increases to higher values at higher relative velocities. Although the absolute numbers suffer from the above discussed spatial filtering problems, it appears that the average line wing ratio of 10 suggested by the observations of Choi et al. (1993) holds for this observations as well.

\subsection{Temperature from $\mathrm{CH}_{3} \mathrm{CN}\left(12_{k}-11_{k}\right)$}

Since we detected 7 lines of the $\mathrm{CH}_{3} \mathrm{CN}\left(12_{k}-11_{k}\right) k$-ladder with $k=0 . .6$, we can utilize the varying excitation levels of the lines with lower level energies $E_{\text {lower }}$ between 58 and $315 \mathrm{~K}$ (see Table 2) to estimate a temperature for the central gas core. We tried to model the whole $\mathrm{CH}_{3} \mathrm{CN}\left(12_{k}-11_{k}\right)$ spectrum toward

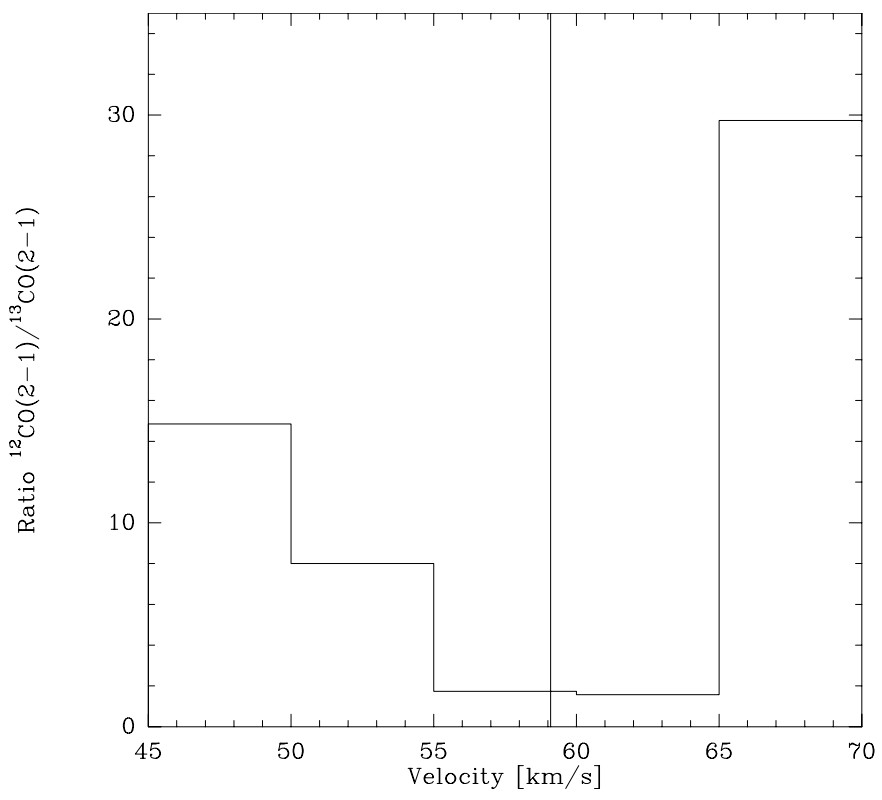

Fig. 11. Ratios between the ${ }^{12} \mathrm{CO}$ and ${ }^{13} \mathrm{CO} J=2-1$ spectra shown in Fig. 7. The ratio increases with increasing velocity of the molecular outflow. We had to truncate the ratio $<45$ and $>70 \mathrm{~km} \mathrm{~s}^{-1}$ because we detect no ${ }^{13} \mathrm{CO}$ signal above the noise at higher velocities. The $v_{\mathrm{lsr}}$ at $59.1 \mathrm{~km} \mathrm{~s}^{-1}$ is marked by a vertical line.

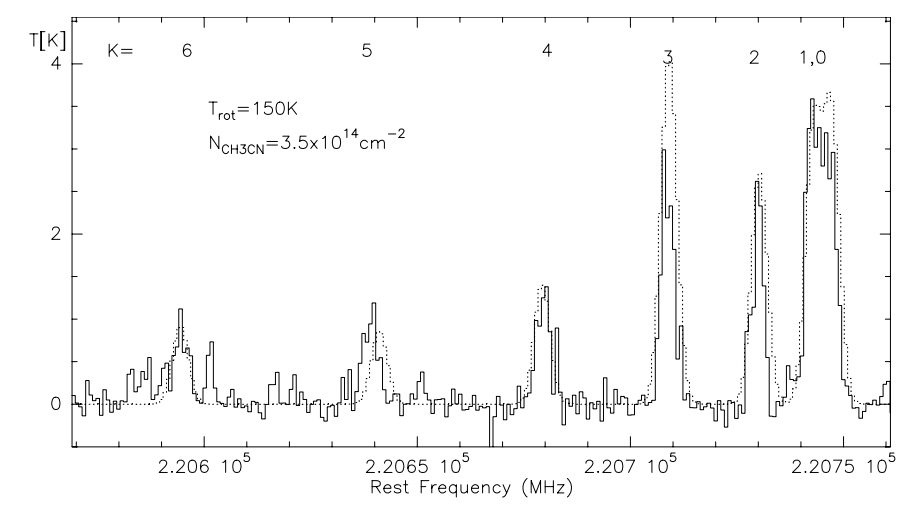

Fig. 12. The full line shows the $\mathrm{CH}_{3} \mathrm{CN}\left(12_{k}-11_{k}\right)$ spectrum toward the $\mathrm{mm}$ continuum position. The dotted line presents a model spectrum with $T_{\text {rot }}=150 \mathrm{~K}$ and $N_{\mathrm{CH}_{3} \mathrm{CN}}=3.5 \times 10^{14} \mathrm{~cm}^{-2}$.

the mm continuum peak position in the local thermodynamic equilibrium using the XCLASS superset to the CLASS software developed by Peter Schilke (priv. comm.). This software package uses the line catalogs from JPL and CDMS (Poynter \& Pickett 1985; Müller et al. 2001). Figure 12 shows the observed $\mathrm{CH}_{3} \mathrm{CN}\left(12_{k}-11_{k}\right)$ spectrum toward the mm peak position and a model spectrum with a temperature of $150 \mathrm{~K}$. This model spectrum fits the observations reasonably well. The main difference is that the model spectrum is optically thin whereas the lower line intensity of the observed $k=3$ line indicates moderate optical depth of the $\mathrm{CH}_{3} \mathrm{CN}$ lines. Nevertheless, the observations clearly show that a hot molecular core has formed at the center of IRAS 18182-1433.

\subsection{Disk signatures?}

Although these observations were meant to study the molecular outflow and thus have not extremely high angular resolution (3" correspond to approximately $13500 \mathrm{AU}$ at the assumed near 

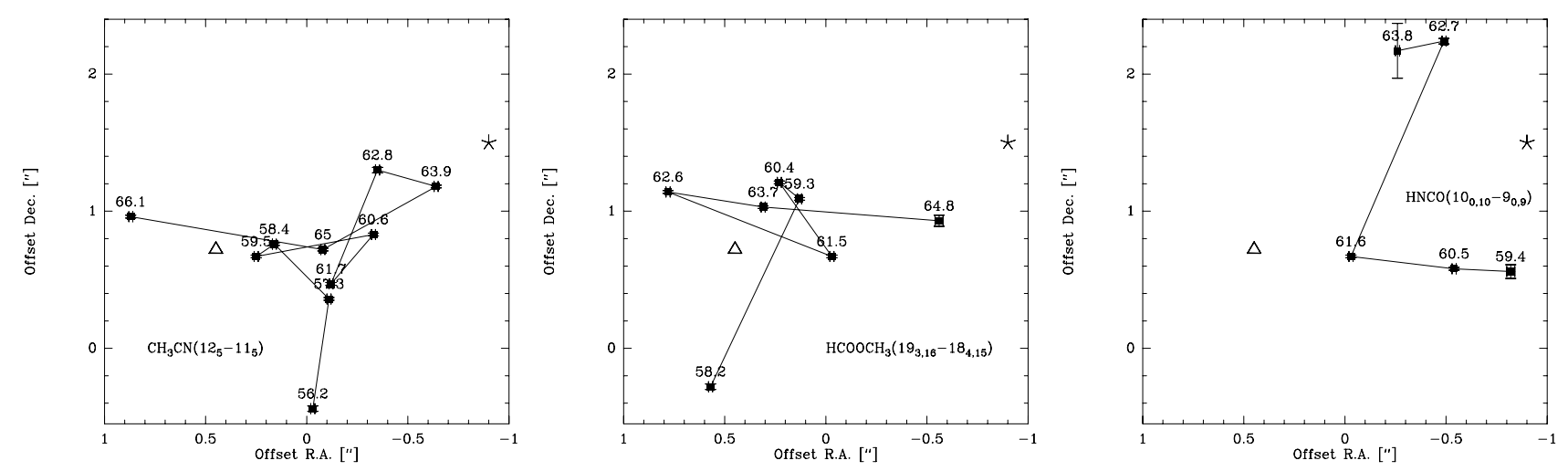

Fig. 13. Positions of different velocity channels for $\mathrm{CH}_{3} \mathrm{CN}\left(12_{5}-11_{5}\right)$, $\left.\mathrm{HCOOCH}_{3}\left(19_{3,16}\right)-18_{4,15}\right)$ and $\mathrm{HNCO}\left(10_{0,10}-9_{0,9}\right)$. The positions were fitted from the imaged data-cubes with the task IMFIT in MIRIAD. The numbers label the center velocities of each channel, and the error-bars are only the statistical errors of the fitting routine. The star and open triangle mark the positions of the mm continuum peak and the $\mathrm{cm}$ source with positive spectral index, respectively. The $0 / 0$ position is the phase reference center. We connected the positions with increasing velocities to stress their random distribution.

kinematic distance), we investigated the typical high-density hot-core tracing molecules $\left(\mathrm{CH}_{3} \mathrm{CN}, \mathrm{HCOOCH} 3, \mathrm{HNCO}\right)$ for potential disk-signatures in their velocity structures. We fitted the peak position of each velocity channel separately to achieve a higher positional accuracy down to $0.5 \mathrm{HPBW} /(\mathrm{S} / \mathrm{N})$ (Reid et al. 1988) and plotted their positions in Fig. 13. We cannot identify any coherent velocity structure in these molecular lines, the velocity distributions appear to be random and are offset from the mm continuum peak. For $\mathrm{CH}_{3} \mathrm{CN}$ and $\mathrm{HCOOCH}_{3}$, the distributions appear somehow in-between the main mm continuum peak and the $\mathrm{cm}$ feature.

\section{Discussion and conclusions}

The combined SMA+IRAM $30 \mathrm{~m} \quad \mathrm{CO}(2-1)$ and the VLA $\mathrm{SiO}(1-0)$ data reveal a multiple outflow system emanating from IRAS 18182-1433. One of the two bipolar outflows traced by the $\mathrm{CO}(2-1)$ emission shows a collimated blue and a cone-like red outflow wing. The northern $\mathrm{SiO}(1-0)$ emission is suggestive of an additional third outflow emanating toward the north. We do not identify a clear southern bipolar counterpart of this potential third outflow. Similar to this, a few other examples have recently been reported where the $\mathrm{SiO}$ emission is tracing only one lobe of an outflow from a massive starforming region (e.g., IRAS 18089-1732, Beuther et al. 2004a; IRAS 18566+0408, Zhang et al. 2006). Although subject to large uncertainties, an analysis of the ${ }^{12} \mathrm{CO}(2-1) /{ }^{13} \mathrm{CO}(2-1)$ line ratios indicates that the $\mathrm{CO}$ line wing opacities decrease with increasing velocities with respect to the $v_{\mathrm{lsr}}$.

All molecular line maps show strong emission approximately 1-2" south-east of the mm continuum peak, well correlated with a cm emission feature maybe due to an underlying thermal jet (Zapata et al. 2005). The offset between the highdensity tracing molecular lines like $\mathrm{CH}_{3} \mathrm{CN}$ or $\mathrm{HCOOCH}_{3}$ and the $1.3 \mathrm{~mm}$ continuum peak is surprising and mainly two possibilities are likely to explain that observational feature. First, the $\mathrm{cm}$ emission feature pinpoints the location of a secondary source which remains unresolved in our mm observations (see, e.g., IRAS 16293-2422, Chandler et al. 2005). This secondary source then could be in an evolutionary more evolved state altering the chemistry compared to the potentially younger source embedded at the location of the mm continuum peak. Contrary to that, the $\mathrm{cm}$ emission could be solely due to the jet itself and not be associated with another protostellar object (see, e.g.,
IRAS 16547-4247, Rodríguez et al. 2005). This would suggest that the chemistry is strongly altered by the molecular outflow, which is particularly surprising for typical hot core and dense gas tracers. Only higher angular resolution (sub)mm continuum observations will be capable to resolve this ambiguity.

Although our spatial resolution of $3.6^{\prime \prime} \times 2.4^{\prime \prime}$ corresponds at the assumed near kinematic distance of $4.5 \mathrm{kpc}$ only to an average linear resolution of $\sim 13500 \mathrm{AU}$, it is interesting to note that we see no sub-clustering in the $1.3 \mathrm{~mm}$ continuum data but only one single-peaked source. This is similar to the cases of IRAS $20126+4104$ (Cesaroni et al. 1999) or IRAS 18089-1732 (Beuther et al. 2005b), but it is different to the fragmentation of the core in IRAS 19410+2336 (Beuther \& Schilke 2004). However, one has to keep in mind that the observations toward IRAS $19410+2336$ had a linear spatial resolution of $2000 \mathrm{AU}$, approximately 6 times better than the new data toward IRAS 18182-1433. Therefore, it is too early to constrain differences and similarities between the fragmentation processes of varying cores, but as pointed out in Beuther et al. (2005b), there are potentially different fragmentation signatures in young massive gas cores which are worth further investigation. The identification of three molecular outflows as well as the two cm emission features observed in the vicinity of the mm peak implies the existence of multiple driving sources within this central massive star-forming core. It would be interesting to identify and characterize the driving sources of each outflow independently, but with the given data so far we cannot set further constrains on the individual driving sources. Again higher angular resolution observations at (sub)mm as well as mid-infrared wavelengths are necessary for a more accurate characterization of the driving source properties.

Finding the right molecular line transitions to study massive accretion disks remains a difficult task. The often used molecule $\mathrm{CH}_{3} \mathrm{CN}$ apparently does not work for IRAS $18182-1433$, but the recently suggested $\mathrm{HCOOCH}_{3}$ does not trace any rotating structure in this source as well. While in principle it is possible that there is no rotating gas structure in IRAS 18182-1433, we consider this as unlikely because molecular outflows require accretion and rotating accretion disks (e.g., Cesaroni et al. 2006). Therefore, we believe that the non-detection of any rotation signature close to the core center is mainly an observational problem. Various explanations are possible in such very young massive and dense molecular cores, the main four are: (i) the molecular lines are usually not only produced in 
accretion-disk-like rotating structures but also in their surrounding cores, which results in various overlapping velocity components and no coherent projected 2-dimensional velocity signature. (ii) In many cases, the spatial resolution is still not sufficient and multiple unresolved sources distort the observed velocity field. (iii) Many molecular lines, even the high-density tracer, are strongly affected by the molecular outflow distorting the observed velocity field. (iv) We are dealing with extremely high gas column densities and thus potentially high opacities. This way, one would not even trace the inner rotating structure. These observations confirm that the quest for the best disktracing molecular line transitions is still open.

Acknowledgements. Thank you very much to Peter Schilke for providing the XCLASS software to model the $\mathrm{CH}_{3} \mathrm{CN}$ spectra. Thanks a lot also to Yuan Chen for providing the integrated VLA $\mathrm{SiO}(1-0)$ image prior to publication. H.B. acknowledges financial support by the Emmy-Noether-Program of the Deutsche Forschungsgemeinschaft (DFG, grant BE2578).

\section{References}

Arce, H., Shepherd, D., Gueth, F., et al. 2006, [arXiv: astro-ph/0603071]

Beuther, H., \& Schilke, P. 2004, Science, 303, 1167

Beuther, H., Schilke, P., Menten, K. M., et al. 2002a, ApJ, 566, 945

Beuther, H., Schilke, P., Sridharan, T. K., et al. 2002b, A\&A, 383, 892

Beuther, H., Walsh, A., Schilke, P., et al. 2002c, A\&A, 390, 289

Beuther, H., Hunter, T. R., Zhang, Q., et al. 2004a, ApJ, 616, L23

Beuther, H., Schilke, P., \& Wyrowski, F. 2004b, ApJ, 615, 832

Beuther, H., Zhang, Q., Greenhill, L. J., et al. 2005a, ApJ, 632, 355

Beuther, H., Zhang, Q., Sridharan, T. K., \& Chen, Y. 2005b, ApJ, 628, 800

Cabrit, S., \& Bertout, C. 1990, ApJ, 348, 530

Cabrit, S., \& Bertout, C. 1992, A\&A, 261, 274

Cesaroni, R., Felli, M., Testi, L., Walmsley, C. M., \& Olmi, L. 1997, A\&A, 325, 725

Cesaroni, R., Felli, M., Jenness, T., et al. 1999, A\&A, 345, 949

Cesaroni, R., Galli, D., Lodato, G., Walmsley, C., \& Zhang, Q. 2006, [arXiv:astro-ph/0603093]

Chandler, C. J., Brogan, C. L., Shirley, Y. L., \& Loinard, L. 2005, ApJ, 632, 371

Choi, M., Evans, N. J., \& Jaffe, D. T. 1993, ApJ, 417, 624

De Buizer, J. M., Radomski, J. T., Telesco, C. M., \& Piña, R. K. 2005, ApJS, 156,179

Faúndez, S., Bronfman, L., Garay, G., et al. 2004, A\&A, 426, 97

Forster, J. R., \& Caswell, J. L. 1999, A\&AS, 137, 43
Frerking, M. A., Langer, W. D., \& Wilson, R. W. 1982, ApJ, 262, 590

Garay, G., Brooks, K. J., Mardones, D., \& Norris, R. P. 2003, ApJ, 587, 739

Gibb, A. G., Hoare, M. G., Little, L. T., \& Wright, M. C. H. 2003, MNRAS, 339, 1011

Gueth, F., Bachiller, R., \& Tafalla, M. 2003, A\&A, 401, L5

Hildebrand, R. H. 1983, QJRAS, 24, 267

Hill, T., Burton, M., Minier, V., et al. 2005, MNRAS, accepted [arXiv: astroph/0506402]

Hunter, T. R., Churchwell, E., Watson, C., et al. 2000, AJ, 119, 2711

Klein, R., Posselt, B., Schreyer, K., Forbrich, J., \& Henning, T. 2005, ApJS, 161, 361

Levreault, R. M. 1988, ApJ, 330, 897

Müller, H. S. P., Thorwirth, S., Roth, D. A., \& Winnewisser, G. 2001, A\&A, 370, L49

Molinari, S., Brand, J., Cesaroni, R., \& Palla, F. 1996, A\&A, 308, 573

Molinari, S., Brand, J., Cesaroni, R., Palla, F., \& Palumbo, G. G. C. 1998, A\&A, 336, 339

Molinari, S., Brand, J., Cesaroni, R., \& Palla, F. 2000, A\&A, 355, 617

Mueller, K. E., Shirley, Y. L., Evans, N. J., \& Jacobson, H. R. 2002, ApJS, 143, 469

Ossenkopf, V., \& Henning, T. 1994, A\&A, 291, 943

Plume, R., Jaffe, D. T., \& Evans, N. J. 1992, ApJS, 78, 505

Plume, R., Jaffe, D. T., Evans, N. J., Martin-Pintado, J., \& Gomez-Gonzalez, J. 1997, ApJ, 476, 730

Poynter, R. L., \& Pickett, H. M. 1985, Appl. Opt., 24, 2235

Reid, M. J., Schneps, M. H., Moran, J. M., et al. 1988, ApJ, 330, 809

Rodríguez, L. F., Garay, G., Brooks, K. J., \& Mardones, D. 2005, ApJ, 626, 953

Sault, R. J., Teuben, P. J., \& Wright, M. C. H. 1995, in Astronomical Data Analysis Software and Systems IV, ASP Conf. Ser., 77, 433

Scoville, N. Z., Carlstrom, J. E., Chandler, C. J., et al. 1993, PASP, 105, 1482

Shepherd, D. S., Yu, K. C., Bally, J., \& Testi, L. 2000, ApJ, 535, 833

Shirley, Y. L., Evans, N. J., Young, K. E., Knez, C., \& Jaffe, D. T. 2003, ApJS, 149,375

Sridharan, T. K., Beuther, H., Schilke, P., Menten, K. M., \& Wyrowski, F. 2002, ApJ, 566, 931

Su, Y., Zhang, Q., \& Lim, J. 2004, ApJ, 604, 258

Walsh, A. J., Burton, M. G., Hyland, A. R., \& Robinson, G. 1998, MNRAS, 301, 640

Walsh, A. J., Macdonald, G. H., Alvey, N. D. S., Burton, M. G., \& Lee, J.-K. 2003, A\&A, 410, 597

Wyrowski, F., Schilke, P., Walmsley, C. M., \& Menten, K. M. 1999, ApJ, 514, L43

Zapata, L., Rodriguez, L., Ho, P., Beuther, H., \& Zhang, Q. 2005, ArXiv Astrophysics e-prints

Zhang, Q., Hunter, T. R., \& Sridharan, T. K. 1998, ApJ, 505, L151

Zhang, Q., Sridharan, T. K., Hunter, T. R., et al. 2006, ApJ, submitted 